\title{
Electromagnetic Isolation and Electrical Protection in Spacecraft General Assembly
}

\author{
Xu Botao ${ }^{1}$, Fang Zhikai ${ }^{1}$, Zhang Bin ${ }^{2}$, Lv Jinghui ${ }^{2}$ and Liu Yugang ${ }^{2}$ \\ ${ }^{1}$ Beijing Institute of Spacecraft Environment Engineering, Beijing, China \\ ${ }^{2}$ Beijing Engineering Research Centre of the Intelligent Assembly Technology and Equipment for Aerospace Product, Beijing, \\ China
}

\begin{abstract}
Spacecraft is always equipotential connected. Faults in grounding cause electromagnetic dysfunction for airborne equipment or subsystems, which can be fatal for a satellite mission. Consequences of electromagnetic problems were evaluated in his paper on system level, and typical scenarios of electromagnetic interference were analysed, based on which a recommended system grounding model was put forward. In the end, measures for electromagnetic isolation and protection in spacecraft general assembly were introduced and analysed.
\end{abstract}

\section{Introduction}

In the conventional spacecraft design system, the design of electrical protection and electromagnetic compatibility mostly exists in the stage of principle design, and has little influence on the manufacturing process of electrical and electronic devices. In the assembly stage of spacecraft system, the principle of electrical protection and electromagnetic compatibility is more difficult to implement because of many restrictions. To some extent, this leads to the following conditions: the verification of spacecraft electrical performance depends too much on the electrical performance test, and the verification of spacecraft electromagnetic performance depends too much on the special tests such as EMC, ESD or magnetic test. First, the design and coverage of various test and test conditions are often unrealistic. On the other hand, the verification effect of various tests are not easy to be guaranteed.

Therefore, in spacecraft assembly and testing (AIT) phase, the implementation of mechanical, electrical and thermal control and other aspects, it is necessary for electrical isolation and electromagnetic compatibility factors into consideration, in order to prevent the abnormal function of the spacecraft in a particular state (Chen., 2007).

\section{Significance of Electromagnetic Isolation}

Depending on the mission, the spacecraft can be divided into several subsystems. The electromagnetic characteristics of sensitive system generally includes power system, circuit system, control system, integrated electronic subsystem, control subsystem and payload; while the main electromagnetic interference (EMI) general system including power system, thermal control system, integrated electronic system, payload etc. EMI; common fault source and fault cases are shown in serious cases (Harland and Lorenz, 2005).

The instrument mounting panel of the spacecraft focuses on electronic equipment and cable harness, highpower radiation signal source and high electromagnetic sensitivity components. In order to make them not interfere with each other and coordinate their work, effective EMC design must be carried out. In addition, in the state of the spacecraft life cycle, it is also necessary to avoid local abnormal discharge. Statistical data show that spacecraft faults caused by circuits and electromagnetic problems account for more than $50 \%$ of the total number of spacecraft faults. Therefore, the electrical isolation and electromagnetic protection of spacecraft are of great significance.

The correct grounding design can avoid the abnormal electrical conduction between the different parts of the satellite, and avoid the change of the characteristic impedance of the RF device under the electric and thermal induction, so that the spacecraft can be protected from electromagnetic interference. The correct operation method of spacecraft AIT is also very important, for example, the laying method of cable network and the choice of grounding network topology, etc., and also have the key influence on the electrical performance and electromagnetic performance of spacecraft.

The correct grounding structure is the primary factor for achieving good electromagnetic compatibility. The 
main purpose of grounding is to reduce electromagnetic interference (EMI) (NASA-HDBK-4001, 1998) between spacecraft electronic devices and / or subsystems. In addition, there are shielding, floating ground and so on to achieve electromagnetic isolation auxiliary means (NASA-HDBK-4002A, 1999). Electrical communication model in this paper a spacecraft, describes the typical working condition of spacecraft components of conduction and insulation, and summarized on the basis of the implementation method for spacecraft assembly and testing, and gives some examples of electrical protection of electromagnetic compatibility technology.

\section{Model And Case Study}

\subsection{Charge and discharge model of dielectric in spacecraft}

The discharge mechanism of spacecraft in orbit is essentially different from that of electrostatic discharge in the ground. High voltage discharge is the primary cause of the failure of spacecraft sensitive devices (Huang and Chen, 2004). Therefore, it is meaningful to do a unified analysis of the electrical connection structure of a given spacecraft.

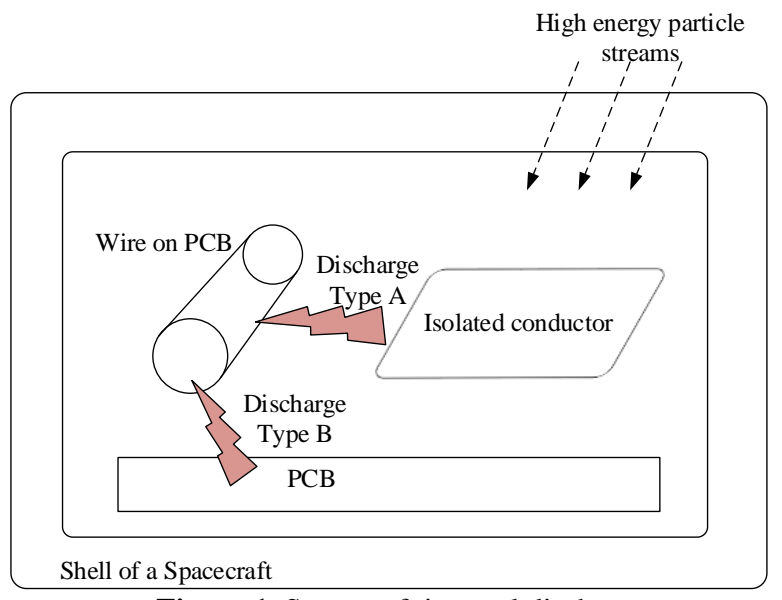

Figure 1. Spacecraft internal discharge

Type A discharge: an accumulation of electrons on an isolated conductor to discharge a circuit.

Type B discharge: an electrical discharge of electrons trapped in a circuit board (dielectric).

When the charging process is simplified and is equivalent to the charging process of $\mathrm{RC}$ circuit, the following equation can be obtained:

$$
\mathrm{E}=\frac{\mathrm{e} \emptyset(0)}{\sigma}\left(1-\exp \left(-\frac{t}{\tau}\right)\right)
$$

Where $\mathrm{E}$ is a medium charging electric field; $\tau$ is the charging time constant, and $\varphi$ stands for the energetic electron flux, $\sigma$ represents conductivity of the dielectric.

According to the formula (1), the maximum electric field that can be reached by deep charging depends on the energy of high energy electrons and the dielectric conductivity of a medium of specified thickness. Therefore, to reduce the possibility and hazard of discharge, in addition to reduce radiation flux by shielding layer, should also be possible to put the whole spacecraft connected into an equipotential body, at the same time try to use high conductivity medium.

It has been pointed out that the maximum charged electric field in a satellite occurs at the junction of the medium with the conductor (spacecraft), so it is important to avoid placing a larger area of non-metallic material near the ESD sensitive device or element (Sheng and Cai, 2007).

At the same time, the study shows that the orbital temperature of the spacecraft surface has a significant influence on the upper limit of the surface charging voltage of spacecraft. The relationship between dielectric conductivity and temperature conforms:

$$
\sigma_{T}=\frac{A}{\mathrm{k} T} \exp \left(-\frac{E_{A}}{k T}\right)
$$

Where $\mathrm{A}$ is a constant determined by the dielectric property; $\mathrm{k}$ is Boltzmann constant, $E_{A}$ is the conductance activation energy of the material; The unit of temperature $\mathrm{T}$ is $\mathrm{K}$ (Kelvin).

Therefore, the control of surface discharge should also refer to the threshold temperature of the thermal control design and the vacuum discharge threshold of the specific dielectric materials and their configurations, In consideration of formula (2) and:

$$
\mathrm{J}=\left(\sigma+\varepsilon \frac{\partial}{\partial \mathrm{t}}\right) \mathrm{E}
$$

It is evident that the higher the temperature, the higher the dielectric conductivity, the smaller the maximum of the surface potential of the dielectric.

\subsection{First Section}

The spacecraft should have a good grounding system to provide a drain path for the accumulated charge generated by the spacecraft interacting with the space environment. The grounding system is characterized by grounding grids throughout the spacecraft. Perfect grounding grid has the following requirements:

First of all, the grounding network shall not form an annular circuit.

When the loop is in relative motion with the spatial magnetic field, the induced current is not sufficient to damage the spacecraft components, but it has a considerable impact on the signal of each subsystem. Similarly, the grounding of low frequency power supply system should follow the principle of single ended grounding.

The basic structure of the spacecraft, any two ends of DC resistance should be a reference limit $(\Omega$ level). Metal structure and the shell of the electronic device should overlap, the overlap resistance should be $\mathrm{m} \Omega$ level.

The power bus and its loop-line should be isolated with the spacecraft ground, bus with potential over DC $42 \mathrm{~V}$ should also have redundant insulation measures. All power buses and loop-lines should be isolated from the equipment shell, with and isolation resistance no less than $1 \mathrm{M} \Omega$.

If the equipment use a secondary power supply, the secondary power wires should also be isolated with each other, the isolation resistance is no less than $1 \mathrm{M}$. The loop- 
lines of the secondary power wires are permitted to connect to the equipment casing.

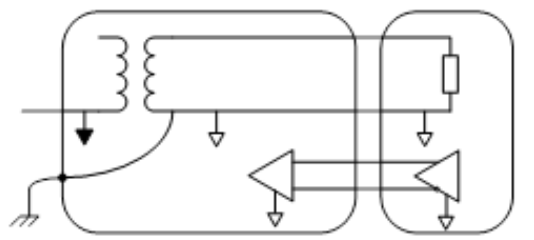

OR

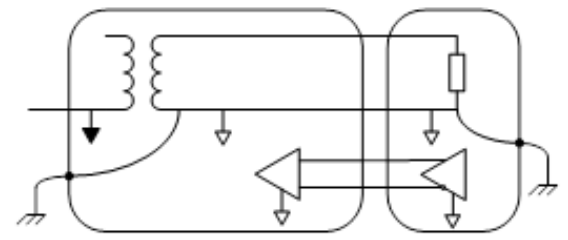

Figure 2. Spacecraft grounding alternatives

\subsection{Grounding for power supply subsystem}

The insulation failure between the spacecraft power system will lead to huge heat release in orbit and endanger the spacecraft's orbit safety. For higher risk failures with insulation, redundant measures are usually adopted to ensure normal operation of the power supply system.

For spacecraft with stable attitude and controllability, the main components of the power supply system include solar array and its drive assemblies, batteries and power distributor. The cables connected to these devices can be defined as the main power cables.

With reference to figure 1 above, an isolated conductor with a certain surface area on the spacecraft should be grounded separately. And furthermore, should the isolated conductor located in a power supply device (such as a solar array drive assembly), a high impedance ground connection method is required. A typical high resistance grounding device consists of 2 parallel resistors. The circuit diagram is shown in figure 3 .

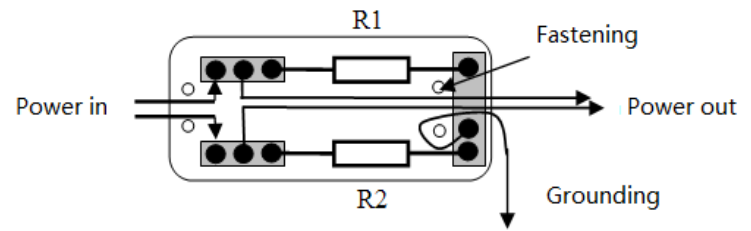

R1、R2: RJ $24-100 \mathrm{k} \Omega / 0.25 \mathrm{~W}$

Figure 3. High resistance grounding circuit

\subsection{Surface potential control in spacecraft assembly}

In the premise of meeting spacecraft's performance, reliability, service life as the whole, if dielectric materials are used on the surface of a spacecraft, such as FR4, Teflon etc., then they must be coated on the surface with conductive membrane, the film resistivity is no more than $1 \times 10^{6} \Omega / \mathrm{m}^{2}$. So as to make them to have a certain capacity of discharging.
By thermal function, the surface of a spacecraft can be divided to two types: heat radiation surface and heat preservation surface.

The heat radiation surface is represented by OSR (Optical solar reflector). In order to meet the requirements of the surface potential control, the surface of OSR should be coated with ITO (Indium-tin Oxide) film, the surface resistivity should be less than $1 \times 10^{6} \Omega / \mathrm{m}^{2}$.

The heat preservation surface is characterized by MLI (Multi-layered insulator). All the multilayer insulation material should be grounded to the zero potential of the spacecraft. Each piece of multilayer insulation material shall be provided with at least two grounding points. Grounding points should be distributed evenly, instead of concentrated in a small area. Each layer of the components should be grounded through the metal foil, and the method is shown in figure 4 .

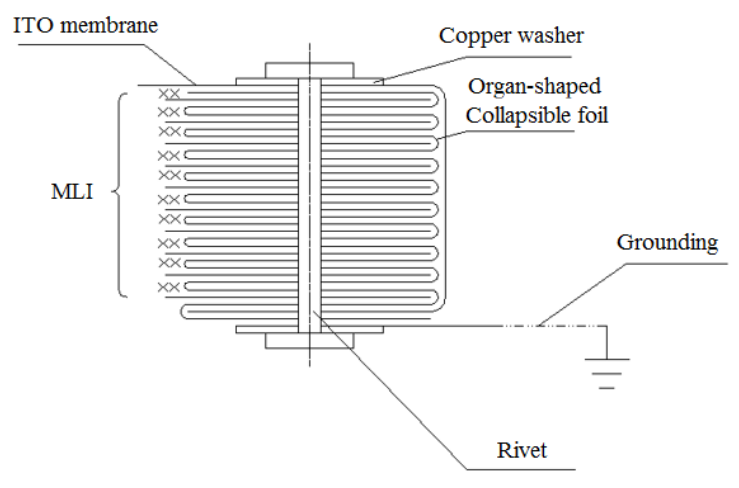

Figure 4. Intersection of Outer MLI Grounding

On one hand, the electronic equipment on spacecraft is sensitive to the EMC, on the other hand it is also an electromagnetic interference source. The overall EMC performance of spacecraft is not only related to the EMC design, but also directly related to the actual shape and path of the assembly. Effective shielding for electromagnetic interference is a practical measure to reduce the coupling of wireless interference, and is also an important way to improve the performance of spacecraft EMC (Liu and Zhou 2015).

In general, there is no need to design and use shielding for uplink or downlink discrete instructions or remote measurements. The signal serial rate is less than a limit value and need not use shielding. If twisted pair wire is use in serial data transmission, the shielding frequency limits can be increased by more than 100 times.

High frequency sensitive signal cable use double-end shield, because single ended cable shielding can only eliminate the capacitive coupling between cables, doubleend shielding can also inhibit the inductive coupling, especially for cable shielding frequency $f_{w}$ is not greater than the interference frequency $\mathrm{f}_{\mathrm{i}}$. So:

$$
f_{w}=\frac{R}{2 \pi L}
$$

Where: $\mathrm{R}$ is the impedance of the cable shielding sleeve, $\mathrm{L}$ is the self-inductance of the shield sleeve at the grounding point, and for the typical cable shield grounding structure, $f_{w}$ is at $\mathrm{kHz}$ level. 


\section{Conclusions}

From the perspective of spacecraft assembly, a spacecraft charging model is proposed. Based on the model, different methods of spacecraft electrical isolation and electromagnetic protection requirements are practiced in the aspect of spacecraft assembly grounding, electrical isolation, surface potential control, electromagnetic shielding and so on. All these is to provide a beneficial experience for the implementation of spacecraft assembly for electromagnetic safety.

\section{References}

1. Chen Shufeng, 2007, Electro-magnetic Technology for Spacecraft, China Science\&Tech Pub., Beijing.

2. David M.Harland and Ralph Lorenz, 2005, Space System Failures. New York: Springer-Verlag.

3. Electrical grounding architecture for unmanned spacecraft, NASA-HDBK -4001 [G], 1998

4. Avoiding problems caused by spacecraft on-orbit internal charging effects, NASA-HDBK -4002A [G], 1999

5. Huang J G, Chen D. A study of deep dielectric charging on satellites by computer simulation. Chinese J. Geophysics, (in Chinese), 2004, 47, (3):392 397

6. SHENG Liyan, CAI Zhenbo. Differential Potential Analysis of GEO Satellite Surface Charging, Spacecraft Engineering, 2007,16(6):93 97

7. Liu Jing, Zhou Liping, Electromagnetic compatibility design and analysis of spacecraft shielded cables. Spacecraft Environment Engineering. 2015(2). Vol. 32, No. 1: 77-82. 